\title{
Melts of Octaacetyl Sucrose as Oral-Modified Release Dosage Forms for Delivery of Poorly Soluble Compound in Stable Amorphous Form
}

\author{
Dorota Haznar-Garbacz, ${ }^{1,7}$ Ewa Kaminska, ${ }^{2}$ Daniel Zakowiecki, ${ }^{3}$ Marek Lachmann, ${ }^{3}$ Kamil Kaminski, ${ }^{4}$ \\ Grzegorz Garbacz, ${ }^{3}$ Przemysław Dorożyński, ${ }^{5}$ and Piotr Kulinowski ${ }^{6}$
}

Received 29 March 2017; accepted 23 October 2017; published online 2 November 2017

Abstract. The presented work describes the formulation and characterization of modified release glassy solid dosage forms (GSDFs) containing an amorphous nifedipine, as a model BCS (Biopharmaceutical Classification System) class II drug. The GSDFs were prepared by melting nifedipine together with octaacetyl sucrose. Dissolution profiles, measured under standard and biorelevant conditions, were compared to those obtained from commercially available formulations containing nifedipine such as modified release (MR) tablets and osmotic release oral system (OROS). The results indicate that the dissolution profiles of the GSDFs with nifedipine are neither affected by the $\mathrm{pH}$ of the dissolution media, type and concentration of surfactants, nor by simulated mechanical stress of biorelevant intensity. Furthermore, it was found that the dissolution profiles of the novel dosage forms were similar to the profiles obtained from the nifedipine OROS. The formulation of GSDFs is relatively simple, and the dosage forms were found to have favorable dissolution characteristics.

KEY WORDS: acetylated saccharides; modified release; sustained release; amorphous drugs; biorelevant dissolution stress test; oral osmotic system.

\section{INTRODUCTION}

The bioavailability of orally administered drugs is very often limited by their solubility. This applies mainly to BCS class II and IV compounds whose overall absorption may be limited by their low solubility in water $(1,2)$. Numerous technological approaches can be applied to overcome this problem. These include processing techniques to improve the

\footnotetext{
${ }^{1}$ Faculty of Pharmacy, Department of Drug Form Technology, Wroclaw Medical University, Borowska 211A, 50-556, Wroclaw, Poland.

${ }^{2}$ Department of Pharmacognosy and Phytochemistry, Medical University of Silesia in Katowice, School of Pharmacy with the Division of Laboratory Medicine in Sosnowiec, ul. Jagiellonska 4, 41-200, Sosnowiec, Poland.

${ }^{3}$ Physiolution GmbH, Walther-Rathenau-Str. 49a, 17489, Greifswald, Germany.

${ }^{4}$ Institute of Physics\&SMCEiBI, University of Silesia, ul 75 Pulku Piechoty 1, 41-500, Chorzow, Poland.

${ }^{5}$ Department of Pharmaceutical Technology and Biopharmaceutics, Pharmaceutical Faculty, Jagiellonian University, ul. Medyczna 9, 30688, Kraków, Poland.

${ }^{6}$ Institute of Technology, Faculty of Mathematics, Physics and Technical Science, Pedagogical University of Cracow, ul. Podchorążych 2, 30-084, Kraków, Poland.

${ }^{7}$ To whom correspondence should be addressed. (e-mail: dorota.haznar-garbacz@umed.wroc.pl)
}

solubilization parameters of the active pharmaceutical ingredient (API). Most commonly applied operations involve the optimization of API particle size or other means of particle engineering, improvement of wetting behavior, use of surfactants, preparation of solid dispersions, and the formulation of self-emulsifying or self-micro-emulsifying systems (3).

Furthermore, the preparation of the APIs in the amorphous form is well known although challenging (4-10). Amorphous substances show altered physico-chemical properties in comparison to their crystalline counterparts (9). Consequently, they often exhibit improved solubility, enhanced dissolution rate, and improved bioavailability (1116). Amorphization can be achieved by various routes, i.e., (i) solvent evaporation, (ii) milling, (iii) freeze- or spray drying, and (iv) quench cooling. These techniques are widely applied in the preparation of immediate release formulations. However, amorphous substances are often labile and therefore stability issues frequently become the major challenge of the formulation efforts. One of the opportunities to stabilize amorphous APIs is the addition of excipients to suppress recrystallization: Examples of materials to suppress recrystallization are polymers and low molecular weight compounds, such as amino acids, polyhydroxy alcohols, traditional as well as modified saccharides

Recently, the importance of acetylated derivatives of saccharides has been emphasized (14-16). These substances are cost-effective and are widely used in the food industry. One example of a modified saccharide is acetylated starch 
(E1451), which is used as a binding agent. Acetylated starch is classified by the Food and Drug Administration (FDA) as a Generally Recognized as Safe (GRAS) material. Numerous investigations regarding the use of various sugar derivatives, i.e., acetylated sucrose, glucose, maltose, galactose, to stabilize amorphous forms of drugs, such as indomethacin, itraconazole, and nifedipine have been published $(6,17,18)$. Solid dispersions composed of amorphous APIs have been obtained by melting with appropriate ratios of active substances and modified saccharides followed by rapid cooling. The binary solid dispersions can be subsequently processed to the final solid dosage forms easily.

Controlled delivery of BCS class II drugs often requires improved solubility and stable drug release for the appropriate time period. Commercially available formulations, e.g., polymer matrix systems with erosion-driven drug release or oral osmotic systems (OROS) with osmotically controlled drug delivery, involve various mechanisms to obtain appropriate drug dissolution. Besides indisputable advantages of such formulations, there are also some drawbacks with regards to high costs and complexity of the systems, limited usefulness for application with poorly soluble APIs, or risk of toxicity increase due to dose dumping (e.g., in the case of coating perforation $(15,19,20))$. For these reasons, there is still need to contemplate novel strategies for controlled delivery of poorly soluble drugs.

Nifedipine (NIF) is a widely used calcium channel blocker, whose efficiency and tolerability have been confirmed in a number of studies (19-21). Both forms of NIF, crystalline and amorphous, are available and the latter one shows significantly improved solubility kinetics and saturation solubility, but it is physically unstable. Numerous researchers have investigated the pharmacokinetics of oral as well as parenteral formulations. It has been demonstrated that a rapid increase of nifedipine plasma concentrations results in an increased occurrence of undesirable effects, e.g., hearing problems $(20,21)$. Consequently, it has become evident that improvement of efficiency and safety of the cardiovascular diseases treatment with nifedipine is required (19).

In a pre-formulation study, nifedipine has been used as a typical BCS class II drug, which could be utilized as model for development of novel techniques of controlled drug delivery (21). The study has outlined the advantages of the application of octaacetyl sucrose for the stabilization of amorphous nifedipine. It has been shown that higher concentrations (> $50 \%$ by weight) of modified disaccharides sufficiently suppress recrystallization. Moreover, it has been found that the amorphous form of API remained stable even after 1 year of storage under room conditions (22). In this context, application of acetylated saccharides in the formulation seems to be a promising option $(11,17,18)$.

To date, the use of acetylated carbohydrates has been focused on the development of immediate release formulations $(21,23)$. However, their application in modified release dosage forms has not been sufficiently investigated.

Therefore, the goals of the study were:

Development and performance assessment of glassy solid dosage forms (GSDFs) - oral matrix modified release dosage forms-for delivery of poorly soluble compound (nifedipine) in stable amorphous form,
- Comparing the developed formulation with commercially available nifedipine products in simulated gastrointestinal tract conditions in vitro and to relate them to in vivo literature data.

The presented study follows pre-formulation investigations performed by the members of our group (22).

\section{Materials and Methods}

\section{Preparation of GSDFs Containing Glassy Nifedipine and Acetylated Sucrose}

Nifedipine reference standard (batch number 516A051), sucrose octaacetate $\left(\mathrm{C}_{28} \mathrm{H}_{38} \mathrm{O}_{19}\right)$, pepsin (USP grade), and pancreatin (USP grade) were supplied by Sigma Aldrich (Steinheim, Germany). High-purity water used in analytical procedures was obtained from a Millipore Milli-Q plus ultrapure water system (Bedford, MA, USA). HPLC grade methanol was purchased from VWR International (Fontenay-sous-Bois, France). All other chemicals and reagents of analytical grade were provided by VWR (Darmstadt, Germany).

Nifedipine and acetylated sucrose (30 and $150 \mathrm{mg}$, respectively, for each single matrix) were weighed and transferred to conical silicone molds and gently mixed. Subsequently, the forms were heated up to $190 \pm 5^{\circ} \mathrm{C}$ in an oil bath (IKA ${ }^{\circledR}$ RCT basic, IKA ${ }^{\circledR}-W e r k e ~ G m b H$, Staufen, Germany) and the powder blends were molten for $10 \mathrm{~min}$ under visual inspection. After melting, the molds were removed from the oil bath and cooled down at room temperature $\left( \pm 22^{\circ} \mathrm{C}\right)$. The conical glassy nifedipine matrices (4 $\mathrm{mm}$ height, $6 \mathrm{~mm}$ diameter at the bottom each) were retrieved, weighed, and stored at room temperature under protection from light.

\section{Reference Commercial Formulations}

For comparison, the following commercial formulations were used: GITS Adalat OROS (Bayer, Berlin, Germany) and Nifedipine Sandoz (Sandoz, Kundl, Austria). Each of the commercial formulations contained $30 \mathrm{mg}$ of the drug, i.e., the same dose as for developed glassy melt formulation.

\section{Solubility of Crystalline Nifedipine and its Amorphous Form from Solid Dispersion with Acetylated Sucrose}

The solubility of crystalline nifedipine and nifedipine in solid dispersions with acetylated sucrose was determined using USP phosphate buffer ( $\mathrm{pH}$ 6.8). For this purpose, the powders $(\sim 10.5 \mathrm{mg})$ were transferred to glass vials $(n=3)$ containing a magnetic stirring bar $(3 \mathrm{~mm}$ in diameter, $7 \mathrm{~mm}$ in length each). After addition of $6 \mathrm{~mL}$ preheated medium $\left(37 \pm 0.5^{\circ} \mathrm{C}\right)$, the samples were stirred for $24 \mathrm{~h}$ at $37 \pm 0.5^{\circ} \mathrm{C}$, $300 \mathrm{rpm}\left(\mathrm{IKA} \AA\right.$ RCT basic, IKA ${ }^{\circledR}-$ Werke GmbH, Staufen, Germany). Samples of 200 to $300 \mu \mathrm{L}$ were withdrawn with a 2-mL polyethylene syringe (Injekt ${ }^{\circledR}$ Solo, B. Braun Melsungen AG, Melsungen, Germany) and filtered through a PTFE-filter (CHROMAFIL $®$ Xtra H-PTFE-20/13, Macherey-Nagel GmbH \& Co. KG, Düren, Germany). 
Samples were taken at 5,10, 15, and $30 \mathrm{~min}$ as well as 1, 2, 3, 4,6 , and $24 \mathrm{~h}$ after addition of dissolution media. Subsequently, $200 \mu \mathrm{L}$ of the supernatant was diluted in $600 \mu \mathrm{L}$ HPLC solvent (methanol: water 50:50, pH 3.0) and transferred to HPLC vials for chromatographic analysis. All procedures described were conducted under light protection to avoid UV degradation of binary mixtures and reference substance.

The concentration of nifedipine in solution was determined using a Merck-Hitachi Elite LaChrom HPLC system (Merck, Darmstadt, Germany) equipped with L-2130 Pump, L-2200 autosampler, L-2400 UV Detector, and a Jetstream II Plus column oven. A LiChrospher 100 RP-18 (5 $\mu \mathrm{m})$ $125 \times 4.0 \mathrm{~mm}$ column (Merck Millipore, Darmstadt, Germany) was used to achieve chromatographic separation. The mobile phase consisted of mixture of methanol and water $(65: 35 v / v)$ was adjusted to $\mathrm{pH} 3.0$ with orthophosphoric acid ( $85 \mathrm{vol} \%$ ). Samples were separated at a flow rate of $0.8 \mathrm{~mL} /$ min and a column temperature of $35^{\circ} \mathrm{C}$. A partial loop with needle overfill option was used, and the injection volume was kept at $50 \mu \mathrm{L}$ for all experiments. The UV detection of NIF was carried out at wavelength $\alpha=238 \mathrm{~nm}$. The acquired data were processed using the Clarity software (Data Apex, Prague, Czech Republic).

\section{Standard Dissolution Tests}

The dissolution tests were performed using a USP apparatus 2 (ERWEKA DT80, ERWEKA GmbH, Heusenstamm, Germany, PT-DT 7, PharmaTest, Hainburg, Germany) at rotational speed of $100 \mathrm{rpm}$ at $37.0 \pm 0.5^{\circ} \mathrm{C}$. The studies of NIF dissolution were carried out in the following dissolution media: (a) $\mathrm{HCl}$ Solution $\mathrm{pH} 1.0$ with $0.1 \% \mathrm{~m} / \mathrm{v}$ SDS (sodium dodecyl sulfate); (b) USP acetate buffer $\mathrm{pH} 4.5$ with $0.1 \% \mathrm{~m} / \mathrm{v}$ SDS; and (c) USP buffer solution for nifedipine extend release tablets $\mathrm{pH} 6.8$.

The amounts of the drug dissolved were determined using online UV-Vis spectroscopy. The samples were filtered through PES (polysulfone) filter with a pore size of $0.8 \mu \mathrm{m}$ (Sartorious, Göttingen, Germany) and analyzed.

Quartz cuvettes of $10 \mathrm{~mm}$ path length (Hellma, Müllheim, Germany) were used with a UV 1602 spectrophotometer (Shimadzu, Duisburg, Germany) and an Agilent 8543 spectrophotometric system (Agilent, Santa Clara, USA) with the photometers set to differential mode at two wavelengths of $\lambda=330 \mathrm{~nm}$ for the NIF signal and $\lambda=450 \mathrm{~nm}$ for the background, respectively.

All dissolution experiments and analytical procedures were performed under light protection. For evaluation of water penetration into the GSDFs the dissolution experiment was repeated with addition of $0.1 \%$ methylene blue into the medium. After $4 \mathrm{~h}$ of experiment, the tablets were withdrawn from the solution and photographed. The images were performed using stereomicroscope OPTA-TECH NZS-810.

\section{Biorelevant Dissolution Stress Test}

Simulation of the mechanical stresses of the maximal physiological intensity was performed using the biorelevant dissolution stress test device. A detailed description of the device is given elsewhere (24). In the following, a brief overview is provided. The dissolution stress test device enables the exposition of the specimens to repeating sequences of movements, pressure waves, and phases of rest as they may act on extended release dosage forms during gastrointestinal transit. The apparatus used in this study was driven in two simple stress sequences of agitation within the boundaries of conditions observed in vivo.

Both sequences comprised of phases of a dynamical stress caused by rotation of the central apparatus axis at $100 \mathrm{rpm}$ over a period of $1 \mathrm{~min}$ followed by three symmetrical pressure waves of $6 \mathrm{~s}$ duration at $300 \mathrm{mbar}$. The stress phases were repeated every $20 \mathrm{~min}$ in case of the first $(19+1)$ sequence and every $60 \mathrm{~min}$ in case of second $(59+1)$ sequence.

Studies of NIF dissolution were carried out in the following separate dissolution media: $\mathrm{HCl}$ solution $\mathrm{pH} 1.0$ with $0.1 \% \mathrm{~m} / \mathrm{v}$ sodium dodecyl sulfate (SDS), USP acetate buffer $\mathrm{pH} 4.5$ with $0.1 \% \mathrm{~m} / \mathrm{v}$ SDS, and USP buffer solution for nifedipine extend release tablets $\mathrm{pH} 6.8$.

The impact of the surfactant concentration on the dissolution of GSDFs was investigated under 19+1 stress sequence using $\mathrm{pH} 6.8$ buffer solution for nifedipine extend release tablets containing 0.5 or $1.0 \%$ of SLS. The impact of physiological surfactants on the dissolution profiles was investigated using fed-state simulated intestinal fluid (FeSSIF).

The amount of the drug dissolved was determined using online UV-Vis spectroscopy as described in the previous section. All dissolution experiments and analytical procedures were performed under light protection.

\section{Processing and Comparison of the Dissolution Profiles}

The dissolution profiles obtained under standard and stress test conditions were processed using commercial software (Axum 5.0, MathSoft, Inc., Cambridge, MA, USA). The mean dissolution profiles obtained under the different test conditions were analyzed using coefficients of the linear regression as well as similarity coefficient (F2).

\section{Robustness of GSDFs Towards Digestive Enzymes}

The impact of pepsin and pancreatin was qualitatively investigated by incubating the GSFDs in $250 \mathrm{~mL}$ of USP simulated gastric fluid test solution (USP SGF TS) and USP simulated intestinal fluid test solution (USP SIF TS). USP phosphate buffer $\mathrm{pH} 6.8$ with $0.1 \%$ surfactant was used as a reference medium allowing the determination of the potential impact of the digestive enzymes on the durability of the SGDFs. The incubation was performed in beakers $(\varnothing 10 \mathrm{~cm}$, $800 \mathrm{~mL}$ volume) using a shaking water bath (Julabo SW22, Julabo GmbH, Seelbach, Germany) at $37^{\circ} \mathrm{C}$ and $30 \mathrm{cpm}$ over $4 \mathrm{~h}$. After each hour of experiment, the tablets were withdrawn from the solution, photographed using a calibrated EOS 60D digital camera (Canon, Tokyo, Japan) and processed using commercial software (Google Nik Collection, Google, Mountain View, USA) 


\section{RESULTS}

GSDFs containing nifedipine and acetylated sucrose were transparent, of yellow color, and had a smooth surface. The photographic representation of the formulation is given in Fig. 1. The GSDFs contained neither pores nor air inclusions, which was confirmed by visual inspections using optical microscopy.

Prepared dosage forms fulfilled the pharmacopoeial requirements concerning uniformity of content and mass uniformity. The NIF content variability was about $0.5 \%$, and the weight variation of the GSDFs did not exceeded $0.3 \%$.

\section{Solubility Study of Crystalline Nifedipine and its Amorphous Form from the Solid Dispersion with Acetylated Sucrose}

The solubility of the crystalline form of nifedipine and nifedipine from the solid dispersion with acetylated sucrose was determined using the USP phosphate buffer ( $\mathrm{pH} 6.8$ ). The results of the solubility study are given in Fig. 2 .

In comparison to the nifedipine reference substance, the solubility of NIF from solid dispersion was enhanced slightly. The crystalline nifedipine reached a saturation concentration of $10 \mu \mathrm{g} / \mathrm{mL}$ within approximately $90 \mathrm{~min}$ of incubation. The saturation solubility was maintained for $6 \mathrm{~h}$. In case of the solid dispersion of amorphous nifedipine with acetylated sucrose, fast dissolution of nifedipine and supersaturation of the drug solution was observed within the first $60 \mathrm{~min}$ of incubation at a concentration of the API corresponding to approximately $17 \mu \mathrm{g} / \mathrm{mL}$. Supersaturation above $16 \mu \mathrm{g} / \mathrm{mL}$ was maintained for $3 \mathrm{~h}$. Subsequently, a slight decrease in the concentration of nifedipine down to approximately $15 \mathrm{mg} / \mathrm{mL}$ occurred. Noticeably, the API concentration achieved at $360 \mathrm{~min}$ with the solid dispersion was still approximately $40 \%$ higher than in the case of crystalline drug. The determined saturation solubilities of nifedipine amounted to approximately 14 and $10 \mu \mathrm{g} / \mathrm{mL}$ for the solid dispersion and crystalline drug, respectively.

\section{Standard Dissolution Tests}

The results of standard dissolution tests are given in the Fig. 3a-c. Dissolution profiles of glassy solid dispersions of nifedipine with acetylated sucrose are shown in Fig. 3a. Dissolution of nifedipine in all media was linear with a constant dissolution rate ranging from 3.9 to $4.1 \% / \mathrm{h}$. The slopes of the dissolution profiles as well as the corresponding coefficients of linear regression determined on mean dissolution profiles are shown in Table I. During the dissolution tests, the formulations did not swell or erode. Moreover, no lag phase was observed. Such a behavior may suggest that the dissolution rate is dependent on the surface area of the investigated formulation. This observation could be of great importance, since it showed that dissolution of nifedipine could be controlled and optimized solely by manipulation of the surface of studied formulations (25).

Dissolution kinetics of the MR tablets showed a significant dependency on the $\mathrm{pH}$ of the dissolution medium as shown in Fig. 3b. The dissolution rate was the fastest at $\mathrm{pH} 6.8$ at which 50 to $80 \%$ of the drug was dissolved approximately after 6 to $8 \mathrm{~h}$. Slower drug release was observed in buffer solutions at $\mathrm{pH} 4.5$, where 50 to $80 \%$ of the active substance was dissolved after approximately 16 to $20 \mathrm{~h}$. At $\mathrm{pH} 1.0$, the dissolution was the slowest and reached a maximum of $50 \%$ within approximately $22 \mathrm{~h}$. Visual inspection performed during the dissolution tests revealed $\mathrm{pH}$-dependent swelling and erosion of the tablets.

The dissolution profiles of Adalat OROS GITS $30 \mathrm{mg}$ in different $\mathrm{pH}$ are given in Fig. 3c. The dissolution profiles of the GITS system show a characteristic lag phase of up to $2 \mathrm{~h}$ in contrast to the dissolution profiles for glassy solid dispersions. The dissolution profiles for all test specimens of the OROS formulation were very similar regardless of the $\mathrm{pH}$ of the medium. Dissolution rate estimated over the entire dissolution profiles, amounted to $4.2 \% / \mathrm{h}$, and complete dissolution of the drug load was achieved within $23 \mathrm{~h}$.

\section{Biorelevant Dissolution Stress Tests}

The results of the biorelevant dissolution experiments performed under stress sequencies (19+1 and 59+1), using USP phosphate buffer for nifedipine ER tablets pH 6.8 are presented in Fig. 4a-b. In the case of nifedipine-acetylated sucrose GSDFs as well as Adalat OROS GITS, the mechanical agitation under both stress regimens did not cause dose dumping of the drug. Similarly to the standard dissolution tests for Adalat OROS GITS, a 2-h lag time was observed for that formulation, while no lag time was witnessed for the developed formulation with acetylated sucrose. The dissolution profiles of both formulations were closely to linear and slightly higher for OROS GITS. The calculated dissolution rates amounted to approximately 4.9 and $4.2 \% / \mathrm{h}$ for Adalat OROS GITS and solid dispersions of nifedipine, respectively. Visual inspection performed during the dissolution stress tests revealed that the nifedipine formulation with modified sucrose was highly resistant to mechanical agitation of physiological range and did not erode or deform under applied mechanical stresses.

In contrast, the dissolution profiles of MR tablets clearly indicated the susceptibility of the matrices to mechanical agitation within the physiological range. Each simulated stress sequence effected rapid erosion of the tablet matrices and following dose dumping. In this case, dissolution was completed within approximately $2.3 \mathrm{~h}$ under $19+1$ sequence (stress phase repeated every $20 \mathrm{~min}$ ) and $4.2 \mathrm{~h}$ under $59+1$ sequence (stress phase repeated every $60 \mathrm{~min})$.

The stress tests of GSDFs performed in dissolution media containing 0.5 and $1 \%$ SLS as well as FeSSIF under $19+1$ stress sequence led to similar dissolution profiles as obtained using USP buffer for nifedipine tablets with $0.1 \%$ SLS. The calculated F2 values were $\geq 93$. The results are given in Fig. 5.

\section{Robustness of GSDFs Towards Digestive Enzymes}

The visual inspection of the GSDFs performed during the incubation in USP phosphate buffer for nifedipine tablets with $0.1 \%$ SLS, USP SGF TS, and USP SIF TS yielded no differences in visual appearance. During the incubation in these dissolution media, the formulations were not digested, 

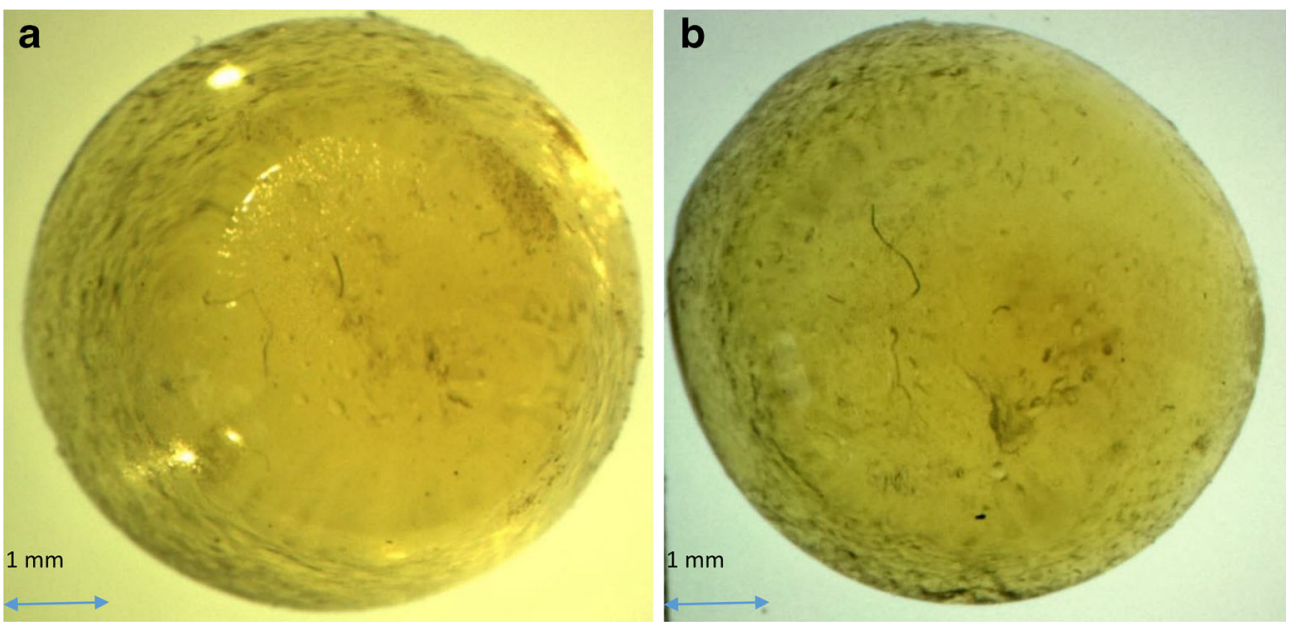

Fig. 1. Photographic representation of the glassy solid dosage forms (GSDFs) with nifedipine: before the dissolution test (a) and at $4 \mathrm{~h}$ of dissolution performed in medium containing $0.1 \%$ blue colorant (b). The scale is given by the insets

apparently did not swell or erode. The results of the visual inspections are given in Fig. 6.

\section{DISCUSSION}

\section{Stability-Result of Pre-Formulation Study}

The comprehensive studies on solid dispersions of NIF with four representative acetylated saccharides, with the use of several experimental methods, such as DSC (differential scanning calorimetry), FTIR (Fourier transform infrared spectroscopy), XRD (X-ray diffraction), BDS (broadband dielectric spectroscopy) as well as molecular dynamic simulations have been carried out in the pre-formulation phase of the study and are described elsewhere (22). It has been shown that in contrast to acetylated monosaccharides, modified disaccharides affect crystallization of active substance both above and below the glass transition temperature $\left(T_{g}\right)$. It has been found that the activation barrier for the crystallization of nifedipine increases significantly in binary mixtures composed of acetylated disaccharides for the experiments carried out above $T_{\mathrm{g}}$. The long-term studies on the physical stability of investigated solid dispersions stored in the close vicinity and below $T_{g}$ revealed that for the higher amount of API, nifedipine tends to re-crystallize from each solid dispersion, while for the higher content of excipient, this process seems to be completely suppressed. It has been suggested that enhanced stability of active substances in binary systems with acetylated maltose and sucrose is most likely due to additional barriers related to (a) intermolecular interactions and/or (b) diffusion of API in the saccharide matrix that must be overcome to trigger the crystallization process. In formulations comprising a saccharide matrix, the API remained stable even after 1 year of storage at room temperature. These results are excellent prerequisite for a formulation study. Thus, it was decided to check whether the GSDFs obtained by co-melting nifedipine and acetylated sucrose investigated in this study are robust with respect to the exposure to media of different $\mathrm{pH}$ as well as mechanical stress of biorelevant intensity.

\section{Dissolution/Release from Surface}

The use of active substances molten/mixed with modified saccharides offers the additional advantage of flexible adjustment of the drug delivery rate in order to meet the therapeutic requirements. During the dissolution tests performed using different setups and dissolution media as well as incubation in solutions of colorants and digestive enzymes, it was observed that the dissolution of nifedipine from GSDFs
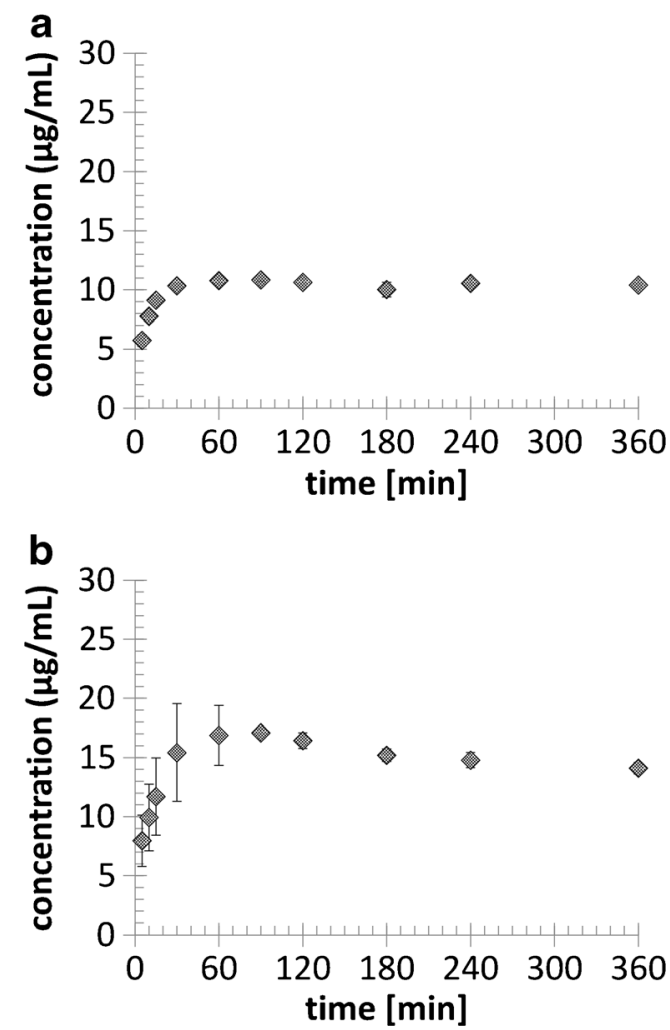

Fig. 2. Results of solubility study of crystalline form of nifedipine (a) and amorphous solid dispersion of nifedipine with acetylated sucrose (b). Given are means of $n=3$; the standard deviation is given by error bars 
a

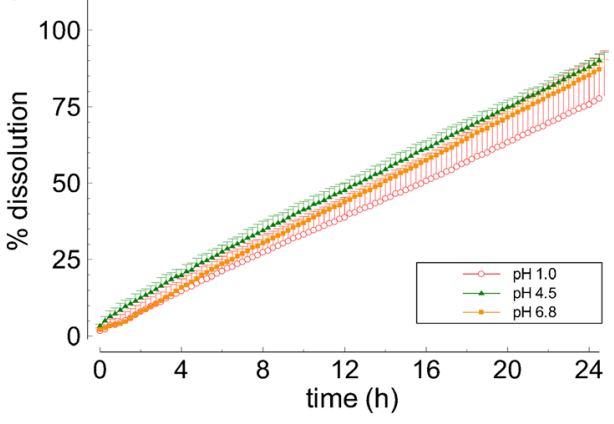

b

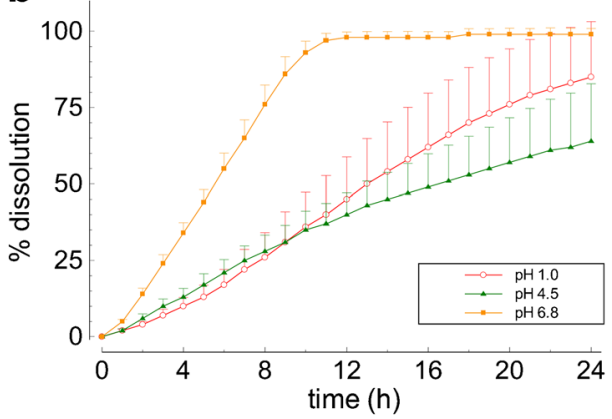

C

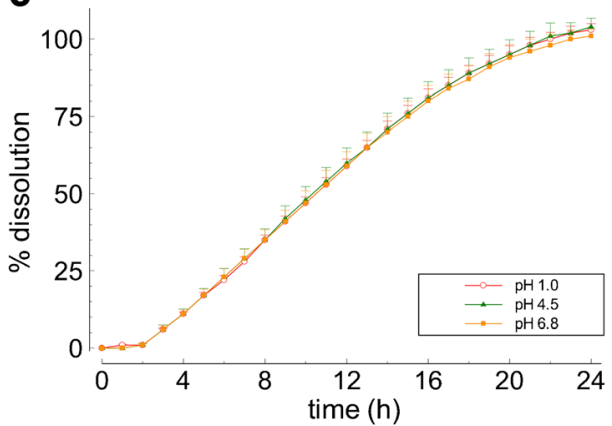

Fig. 3. Dissolution of MR tablets (a), GSDFs-solid dispersions of nifedipine with acetylated sucrose (b), and nifedipine OROS (c) in the USP apparatus 2 at $100 \mathrm{rpm}$. Given are means of $n=6$; the standard deviation is indicated by the error bars. The $\mathrm{pH}$ of the dissolution medium is indicated by the insets

takes place only from the surface of the melts (Figs. 1b and 6). The dissolution characteristics of the GSFDs were independent on the type and concentration of the used a
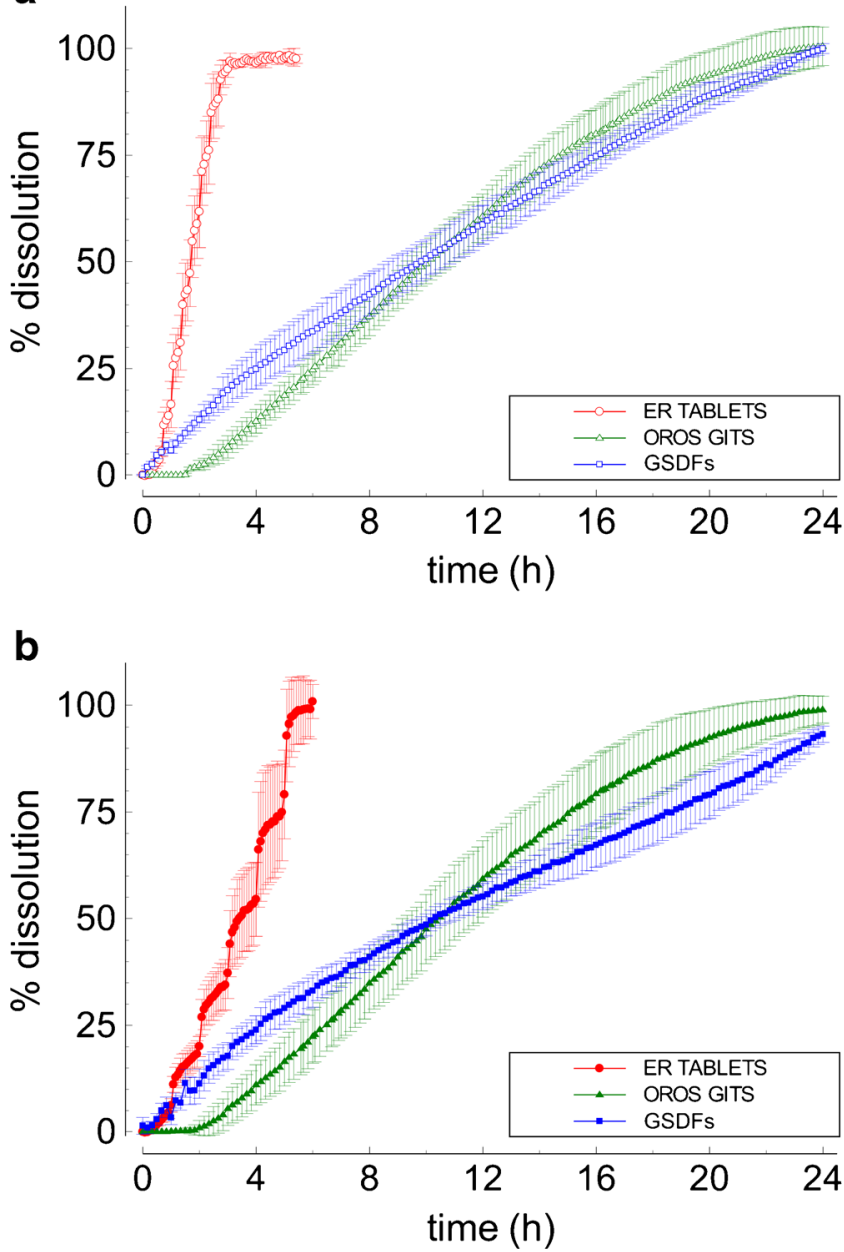

Fig. 4. Dissolution of MR products with nifedipine in the stress test device under stress sequences 19+1 (a) and 59+1 (b) using USP phosphate buffer $\mathrm{pH} 6.8$ with $0.1 \%$ SDS as dissolution medium. Given are means of $n=3$; standard deviation is indicated by the error bars

surfactants (Fig. 5). Therefore, it is likely that dissolution rate of GSDF compositions can be easily adjusted by changing the contact surface area of the binary glassy systems to the dissolution medium by simple means of size and shape modification.

Furthermore, modifications of the dissolution characteristics can be achieved by varying the drug load in the solid

Table I. Linear equations ( $x=\%$ Dissolved $\times \mathrm{Min}^{-1}$ ) and corresponding regression coefficients calculated form the mean dissolution profiles of nifedipine glassy melts obtained under standard and stress test conditions

\begin{tabular}{|c|c|c|c|c|}
\hline \multirow[t]{2}{*}{$\mathrm{pH}$} & \multicolumn{2}{|l|}{ USP apparatus II } & \multirow{2}{*}{$\begin{array}{l}\text { Stress test } \\
\text { Linear equation }\end{array}$} & \multirow[t]{2}{*}{ Regression coefficients $R^{2}$} \\
\hline & Linear equations & Regression coefficients $R^{2}$ & & \\
\hline 1.2 & $y=0.0562 x$ & 0.99797 & n.a. & n.a. \\
\hline 4.5 & $y=0.0642 x$ & 0.98805 & n.a. & n.a. \\
\hline \multirow[t]{4}{*}{6.8} & $y=0.0589 x$ & 0.99879 & Sequence $1(19+1)$ & Sequence $1(19+1)$ \\
\hline & & & $y=0.0601 x$ & 0.99681 \\
\hline & & & Sequence $2(59+1)$ & Sequence $2(59+1)$ \\
\hline & & & $y=0.0597 x$ & 0.9914 \\
\hline
\end{tabular}




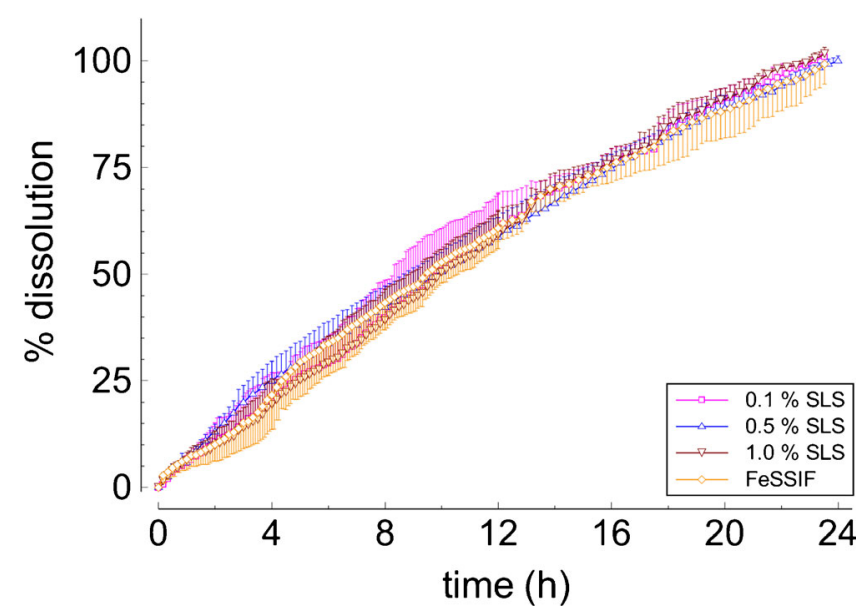

Fig. 5. Dissolution of GSDFs with nifedipine in the stress test device under $19+1$ stress sequence using USP phosphate buffer $\mathrm{pH} 6.8$ with $0.1,0.5$ and $1.0 \%$ SLS as well as FeSSIF dissolution medium. Given are means of $n=3$; standard deviation is indicated by the error bars

dispersion as well as by adjustment of the solubility kinetics of octaacetyl sucrose using alternative excipients. These modifications could be achieved by using other acetylated saccharides, such as acetylated maltose for which the stabilizing effect towards amorphous drug substances has been already proven (22). This will allow for a well-definable and controlled release of the drug, proceeding independently of physiological conditions of the human gastro intestinal passage.

\section{Protection Against Recrystallization}

One of the critical factors limiting the use of amorphous drugs is the hydration of the dosage form. It has been recognized that exposition to moisture or significant humidity levels, prior to dissolution of the API from the dosage form, can trigger recrystallization of amorphous APIs $(26,27)$. Recrystallization not only changes the solubility and dissolution rate but may also alter the delivery kinetics of the dosage form as a whole. Nonetheless, all currently known MR systems require water to be available for polymer swelling or building up osmotic pressure (28).

In the case of ionizable drugs, it may implicate solubility issues which can be at least partially overcome by buffering of the MR matrices or reducing the exposition time by controlling the water penetration into the tablet core during release (27,28). However, with currently available MR systems, the exposition of the drugs to water prior to their release cannot be avoided.

This major challenge can be overcome by the use of nonhydrating systems that facilitate the incorporation of the drugs in molecular dispersions or suspensions. Most desirable matrices should be of low porosity and exhibit very limited and slow water uptake and swelling. Moreover, a dissolution profile should be independent of the $\mathrm{pH}$ and composition of the GI fluids. Thus, such delivery systems would release the active substance only from their surfaces exposed to the GI fluids. Thereby, the exposition time of the drug compound to the aqueous media prior to dissolution would be minimized. Consequently, such systems would bring about advantageous stabilization of the amorphous form of API. So far, a large number of polymeric materials of different water affinity and solubilization behavior is available, including polyvinylpyrolidone (e.g., Kollidon) or polyvinyl caprolactam-polyvinyl acetate-polyethylene glycol graft copolymer (e.g., Soluplus) and others that could be useful for this purpose (23). However, these commonly applied pharmaceutical excipients still bear a risk of water uptake and exposure of the drug substances to the GI liquids prior to dissolution.

Interestingly, the advantageous drug delivery systems characterized by limited or no water sorption during drug delivery can be manufactured using a variety of thermoplastic unit operations, such as melt extrusion, 3D print, or other melt-based technologies using thermostable excipients of desired properties. Owing to their properties saccharides, especially modified ones, seems to be quite an interesting alternative for manufacturing novel dosage forms for

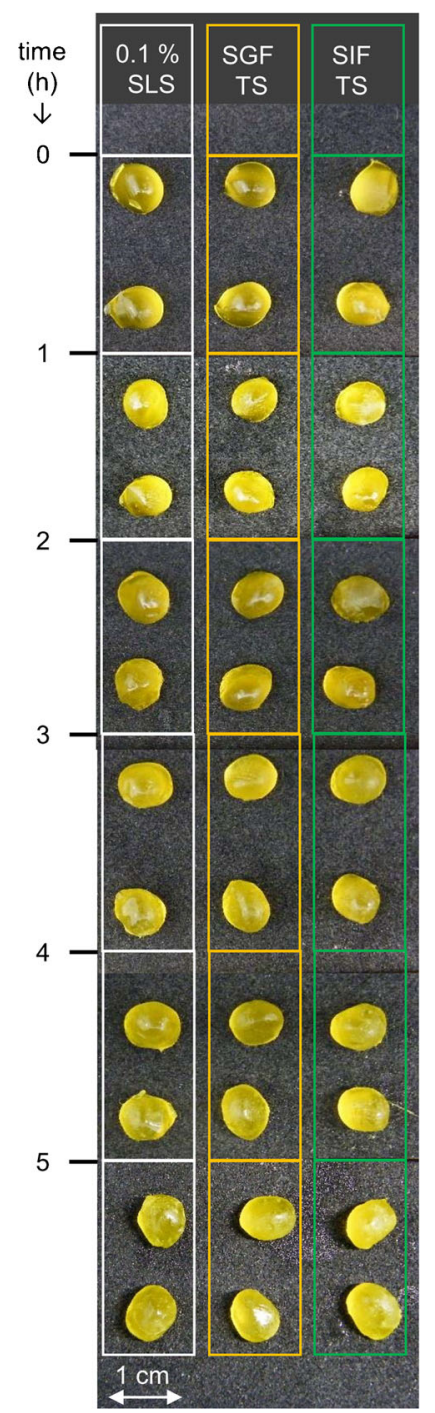

Fig. 6. Photographic representation of the glassy solid dosage forms (GSDFs) with nifedipine during the incubation in USP phosphate buffer $\mathrm{pH} 6.8$ with $0.1 \%$ SLS (0.1\% SLS), USP simulated gastric fluid TS (SGF TS), and USP simulated intestinal fluid TS (SIF TS). The scale and time labels are given by the insets 
controlled delivery of the amorphous forms of poorly water soluble APIs.

\section{Comparison with Commercial ER Systems-Potential Consequences for Pharmacokinetics}

Dissolution profiles obtained under standard and stress conditions were compared with two different types of commercially available MR formulation containing nifedipine. One was a conventional MR tablet-matrix system, of which the drug release is erosion controlled and the other being an osmotic push pull system (OROS).

The pharmacokinetic (PK) properties of one of the OROS systems have been investigated previously under fasted and fed conditions by Schug et al. and Wonnemann et al. The PK profiles obtained for OROS have been reported to be smooth and indicated the absence of sharp plasma peaks or dose dumping events. A lag time of 2 to $3 \mathrm{~h}$ has been observed for this drug product $(29,30)$. This seems to be a distinct characteristic of the OROS formulation. From the biopharmaceutical point of view, such characteristics are very important, especially under fed conditions, when released drug substance can accumulate in the stomach for some time as described by Weitchies et al. $(31,32)$. As reported, the accumulation of released API in the stomach is independent of the dosage form delivery characteristics and occurs mainly in the proximal stomach. In this part of the stomach, tablets may reside for several hours after intake. Due to intragastric mixing and gastric emptying patterns, a large portion of a drug substance that accumulated in the stomach can be transferred into the intestine, thereby causing unexpected, elevated drug plasma levels. According to Weitschies et al. (32), the positive food effects can be related to the intragastric localization and absence of the lag time in the dissolution characteristic of a MR formulation. Such undesired drug delivery behavior has been observed for modified release tablets containing felodipine. However, in the reported case, elevated drug plasma levels have been related to the physiology of gastric emptying, not to the dissolution characteristics of the dosage form itself (31).

Opposed to the OROS, the onset of nifedipine dissolution in vitro from the novel GSDFs appeared immediately after immersion into the dissolution media. Nevertheless, the GSDFs as presented herein could easily be coated with a time-dependent coating to achieve a similar lag time to the OROS system.

The GSDFs are monolithic drug delivery systems and the high variability of gastric emptying times, depending on the prandial conditions as well as food intake, could oppose achievement of the desired release profile. However, owing to the properties of the GSDFs containing octaacetyl sucrose and nifedipine in conjunction with the flexibility of the formulation parameters, this challenge could be met by manufacturing multiparticulate delivery systems. The release profile could then be controlled by adaption of the size and shape of the GSFDs.

Details regarding the pharmacokinetic profiles of the MR tablet formulation included in this study have been reported elsewhere (30). It has been found that in fasted subjects, the maximum nifedipine plasma concentrations were higher after administration of MR tablets than after intake of the OROS formulation. Administration postprandial to a high caloric food - high fat, american breakfast - have yielded an even more profound difference. Up to a threefold increase of the mean $C_{\max }$ has been observed under such conditions in comparison to fasted subjects. On the other hand, food intake has been shown to have no clinically relevant effect on the bioavailability of nifedipine delivered by the OROS GITS. Wonneman et al. have postulated that the observed differences between these two products may have direct therapeutic relevance when substituting a given formulation by the other one (30). This should be particularly taken into consideration if administration conditions are changed in conjunction to the formulation (e.g., fasted to fed-state administration) since the pharmacological and therapeutic actions are closely associated to the drug plasma levels $(19,30)$.

Besides the abovementioned food effects, it has been found that the dissolution profiles of the MR tablets can be also influenced by the $\mathrm{pH}$ as well as mechanical stress of biorelevant intensity. These dissolution characteristics of MR tablets have been reported to bear the potential risk of unwanted drug delivery behavior in vivo. The pharmacokinetic and dissolution characteristics of products having an analogous galenic composition have been previously published $(24,33)$. In contrast, the dissolution of nifedipine from GSDFs was not affected by the $\mathrm{pH}$ of the medium, the type of surfactants and their concentration, or the simulated mechanical stress. In this context, it could be expected that the developed GSDF dosage form would have more reliable in vivo performance (closer to OROS than to matrix tablets). This provides an excellent opportunity to design solid oral dosage forms having stable and predictable nifedipine (and other BCS class II drugs) delivery kinetics in vivo.

\section{CONCLUSIONS}

Previously performed, the pre-formulation studies have proven that modified saccharides such as acetylated sucrose can stabilize the amorphous form of BCS class II drug substances. Based on these findings, amorphous nifedipine solid dispersion with octaacetyl sucrose (GSDFs) were developed. The presented work demonstrated that the use of amorphous binary mixture containing the model, poorly soluble API, nifedipine, and octaacetyl sucrose can be used for preparation of novel oral MR systems. The drug delivery from the novel dosage forms was independent on the dissolution medium $\mathrm{pH}$ and mechanical agitation as well as concentration and type of the added surfactants. The performance of the novel formulation was comparable to the Adalat OROS GITS. However, the manufacturing technology is potentially simpler, robust, reproducible, and easier than in the case of OROS formulations. The novel glassy melt formulation protects amorphous nifedipine from contact with water and subsequent recrystallization. Low variability of the dissolution characteristics as well as independence on $\mathrm{pH}$ and simulated gastric stress are good prerequisites for well-definable drug kinetics in vivo.

\section{ACKNOWLEDGEMENTS}

EK is thankful for the financial support from the National Science Center of Poland based on decision No. 
DEC-2016/22/E/NZ7/00266. DHG and GG kindly acknowledge the financial support of the German General Federal Ministry of Education and Research (BMBF 03IPT612C).

Open Access This article is distributed under the terms of the Creative Commons Attribution 4.0 International License (http://creativecommons.org/licenses/by/4.0/), which permits unrestricted use, distribution, and reproduction in any medium, provided you give appropriate credit to the original author(s) and the source, provide a link to the Creative Commons license, and indicate if changes were made.

\section{REFERENCES}

1. Kasim NA, Whitehouse M, Ramachandran C, Bermejo M, Lennernäs H, Hussain AS, et al. Molecular properties of WHO essential drugs and provisional biopharmaceutical classification. Mol Pharm. 2004;1(1):85-96. https://doi.org/10.1021/mp034006h.

2. Miyaji Y, Fujii Y, Takeyama S, Kawai Y, Kataoka M, Takahashi $\mathrm{M}$, et al. Advantage of the dissolution/permeation system for estimating oral absorption of drug candidates in the drug discovery stage. Mol Pharm. 2016;13(5):1564-74. https:// doi.org/10.1021/acs.molpharmaceut.6b00044.

3. Kumar A, Sahoo SK, Padhee K, Kochar P, Satapathy A, Pathak N. Review on solubility enhancement techniques for hydrophobic drugs. Pharmacie Globale. 2011;3(3):001-7.

4. Yu L. Amorphous pharmaceutical solids: preparation, characterization and stabilization. Adv Drug Deliv Rev. 2001;48(1):2742. https://doi.org/10.1016/S0169-409X(01)00098-9.

5. Kaminski K, Kaminska E, Adrjanowicz K, Grzybowiska K, Wlodarczyk P, Paluch M, et al. Dielectric relaxation study on tramadol monohydrate and its hydrochloride salt. J Pharm Sci. 2010;99(1):94-106. https://doi.org/10.1002/jps.21799.

6. Kaminska E, Adrjanowicz K, Kaminski K, Wlodarczyk P, Hawelek L, Kolodziejczyk K, et al. A new way of stabilization of furosemide upon cryogenic grinding by using acylated saccharides matrices. The role of hydrogen bonds in decomposition mechanism. Mol Pharm. 2013;10(5):1824-35.

7. Boldyrev VV. Mechanochemical modification and synthesis of drugs. J Mater Sci. 2004;39(16):5117-20. https://doi.org/10.1023/ B:JMSC.0000039193.69784.1d.

8. Einfal T, Planinsek O, Hrovat K. Methods of amorphization and investigation of the amorphous state. Acta Pharmaceutica (Zagreb, Croatia). 2013;63(3):305-34. https://doi.org/10.2478/ acph-2013-0026.

9. Dawson KJ, Kearns KL, Yu L, Steffen W, Ediger MD. Physical vapor deposition as a route to hidden amorphous states. Proc Natl Acad Sci U S A. 2009;106(36):15165-70. https://doi.org/ 10.1073/pnas.0901469106.

10. Yadav VB, Yadav AV. Enhancement of solubility and dissolution rate of BCS class II pharmaceuticals by nonaquious granulation technique. Int J Pharm Res Devel. 2010;12:1-12.

11. Adrjanowicz K, Grzybowska K, Kaminski K, Hawelek L, Paluch M, Zakowiecki D. Comprehensive studies on physical and chemical stability in liquid and glassy states of telmisartan (TEL): solubility advantages given by cryomilled and quenched material. Philos Mag. 2011;91(13-15):1926-48. https://doi.org/ 10.1080/14786435.2010.534742.

12. Craig DQ, Royall PG, Kett VL, Hopton ML. The relevance of the amorphous state to pharmaceutical dosage forms: glassy drugs and freeze dried systems. Int J Pharm. 1999;179(2):179207.

13. Qi S, Weuts I, De Cort S, Stokbroekx S, Leemans R, Reading $\mathrm{M}$, et al. An investigation into the crystallisation behaviour of an amorphous cryomilled pharmaceutical material above and below the glass transition temperature. J Pharm Sci. 2010;99(1):196-208. https://doi.org/10.1002/jps.21811.
14. Gupta P, Thilagavathi R, Chakraborti AK, Bansal AK. Role of molecular interaction in stability of celecoxib-PVP amorphous systems. Mol Pharm. 2005;2(5):384-91. https://doi.org/10.1021/ mp050004g.

15. Tobyn M, Brown J, Dennis AB, Fakes M, Gao Q, Gamble J, et al. Amorphous drug-PVP dispersions: application of theoretical, thermal and spectroscopic analytical techniques to the study of a molecule with intermolecular bonds in both the crystalline and pure amorphous state. J Pharm Sci. 2009;98(9):3456-68. https://doi.org/10.1002/jps.21738.

16. Wegiel LA, Mauer LJ, Edgar KJ, Taylor LS. Crystallization of amorphous solid dispersions of resveratrol during preparation and storage-impact of different polymers. J Pharm Sci. 2013;102(1):171-84. https://doi.org/10.1002/jps.23358.

17. Kaminska E, Adrjanowicz K, Tarnacka M, Kolodziejczyk K, Dulski M, Mapesa EU, et al. Impact of inter- and intramolecular interactions on the physical stability of indomethacin dispersed in acetylated saccharides. Mol Pharm. 2014;11(8):2935-47. https://doi.org/10.1021/mp500286b.

18. Kaminska E, Tarnacka M, Kolodziejczyk K, Dulski M, Zakowiecki D, Hawelek L, et al. Impact of low molecular weight excipient octaacetylmaltose on the liquid crystalline ordering and molecular dynamics in the supercooled liquid and glassy state of itraconazole. Eur J Pharm Biopharm: Off J Arbeitsgemeinschaft fur Pharmazeutische Verfahrenstechnik eV. 2014;88(3):1094-104. https://doi.org/10.1016/ j.ejpb.2014.10.002.

19. Toal CB. Formulation dependent pharmacokinetics-does the dosage form matter for Nifedipine? J Cardiovasc Pharmacol. 2004;44(1):82-6.

20. Walley TJ, Heagerty AM, Woods KL, Bing RF, Pohl JE, Barnett DB. Pharmacokinetics and pharmacodynamics of nifedipine infusion in normal volunteers. Br J Clin Pharmacol. 1987;23(6):693-701. https://doi.org/10.1111/j.13652125.1987.tb03103.x.

21. Soons PA, Schoemaker HC, Cohen AF, Breimer DD. Intraindividual variability in nifedipine pharmacokinetics and effects in healthy subjects. J Clin Pharmacol. 1992;32(4):324-31. https://doi.org/10.1002/j.1552-4604.1992.tb03843.x.

22. Kaminska E, Tarnacka M, Wlodarczyk P, Jurkiewicz K, Kolodziejczyk K, Dulski M, et al. Studying the impact of modified saccharides on the molecular dynamics and crystallization tendencies of model API nifedipine. Mol Pharm. 2015;12(8):3007-19. https://doi.org/10.1021/ acs.molpharmaceut.5b00271.

23. Qian F, Huang J, Hussain MA. Drug-polymer solubility and miscibility: stability consideration and practical challenges in amorphous solid dispersion development. J Pharm Sci. 2010;99(7):2941-7. https://doi.org/10.1002/jps.22074.

24. Garbacz G, Wedemeyer RS, Nagel S, Giessmann T, Monnikes $\mathrm{H}$, Wilson $\mathrm{CG}$, et al. Irregular absorption profiles observed from diclofenac extended release tablets can be predicted using a dissolution test apparatus that mimics in vivo physical stresses. Eur J Pharm Biopharm: Off J Arbeitsgemeinschaft fur Pharmazeutische Verfahrenstechnik eV. 2008;70(2):421-8.

25. Daniel Bar-Shalom KN, Clive Wilson. An evaluation of the roles of erodible systems in the oral drug delivery toolbox: the importance of geometry in therapeutic delivery tablets \& capsules. 2012; September:32-9.

26. Colombo P, Bettini R, Peppas NA. Observation of swelling process and diffusion front position during swelling in hydroxypropyl methyl cellulose (HPMC) matrices containing a soluble drug. J Control Release. 1999;61(1-2):83-91. https://doi.org/ 10.1016/S0168-3659(99)00104-2.

27. Colombo P, Bettini R, Santi P, Peppas NA. Swellable matrices for controlled drug delivery: gel-layer behaviour, mechanisms and optimal performance. Pharm Sci Technol Today. 2000;3(6):198-204. https://doi.org/10.1016/S1461-5347(00)002698.

28. Al-Taani BM, Tashtoush BM. Effect of microenvironment $\mathrm{pH}$ of swellable and erodable buffered matrices on the release characteristics of diclofenac sodium. AAPS PharmSciTech. 2003;4(3):110-5. https://doi.org/10.1208/pt040343.

29. Schug BS, Brendel E, Wolf D, Wonnemann M, Wargenau M, Blume HH. Formulation-dependent food effects demonstrated 
for nifedipine modified-release preparations marketed in the European Union. Eur J Pharm Sci: Off J Eur Fed Pharm Sci. 2002;15(3):279-85.

30. Wonnemann M, Schug B, Schmucker K, Brendel E, van Zwieten PA, Blume H. Significant food interactions observed with a nifedipine modified-release formulation marketed in the European Union. Int J Clin Pharmacol Ther. 2006;44(1):38-48.

31. Weitschies W, Friedrich C, Wedemeyer RS, Schmidtmann M, Kosch O, Kinzig M, et al. Bioavailability of amoxicillin and clavulanic acid from extended release tablets depends on intragastric tablet deposition and gastric emptying. Eur J Pharm Biopharm: Off J Arbeitsgemeinschaft fur Pharmazeutische
Verfahrenstechnik eV. 2008;70(2):641-8. https://doi.org/10.1016/ j.ejpb.2008.05.011.

32. Weitschies W, Wedemeyer R-S, Kosch O, Fach K, Nagel S, Söderlind $\mathrm{E}$, et al. Impact of the intragastric location of extended release tablets on food interactions. J Control Release. 2005;108(2-3):375-85. https://doi.org/10.1016/ j.jconrel.2005.08.018.

33. Garbacz G, Blume $H$, Weitschies W. Investigation of the dissolution characteristics of nifedipine extended-release formulations using USP apparatus 2 and a novel dissolution apparatus. Dissolut Technol. 2009;16:7-13. 